\title{
Pulmonary function, exercise performance, and growth in survivors of congenital diaphragmatic hernia
}

\author{
S S Marven, C M Smith, D Claxton, J Chapman, H A Davies, R A Primhak, C V E Powell
}

\begin{abstract}
A cohort of survivors of congenital diaphragmatic hernia (CDH), with matched controls, was studied to assess growth, respiratory function, and exercise performance. Nineteen of $\mathbf{2 4}$ survivors from an 11 year period $(79 \%)$ were compared with 19 matched controls. Subjects had detailed auxology, performed spirometry and cycle ergometry, and completed questionnaires about respiratory symptoms and exercise. There were no significant differences between the groups for height, weight, sitting height, head circumference, or body mass index expressed as SD scores. The mean $(95 \%$ confidence interval) percentage predicted forced vital capacity (FVC) was $84.7 \%(79.1$ to 90.3$)$ in index cases and $96.5 \%(91.4$ to 101.6$)$ in controls $(p<0.01)$. There was no significant difference in total lung capacity. Expiratory flow rates corrected for FVC were also similar between groups, suggesting normal airway function relative to lung size. Mean maximum oxygen consumption in $\mathrm{ml} / \mathrm{kg} / \mathrm{min}$ was 40.1 (36.8 to 43.4) and 42.2 (38.5 to 45.8$)$ in index and control cases. These differences were not significant. Index cases achieved a similar minute ventilation to controls by more rapid and shallower breathing. Index cases had lower perception of their own fitness and lower enjoyment of exercise, although habitual activity levels were similar. Survivors of $\mathrm{CDH}$ repair have reduced functional lung volumes, but normal airway function compared with matched controls. They have no growth impairment nor significant impairment of exercise performance, although they have more negative perceptions of their own fitness. They should be encouraged and expected to participate fully in sport and exercise.

(Arch Dis Child 1998;78:137-142)
\end{abstract}

Keywords: congenital diaphragmatic hernia; exercise performance; growth; spirometry

Sport and Exercise Laboratory, Sheffield Hallam University D Claxton

J Chapman

Correspondence to: Dr C V E Powell, Sheffield Children's Hospital, Western Bank, Sheffield S10 2TH.

Accepted

6 September 1997 significant mortality from pulmonary hypoplasia and from associated malformations. ${ }^{1}$ Although there is evidence of persisting parenchymal abnormalities in the lungs of survivors, ${ }^{2-7}$ their functional impact is unclear. Reports of lung function in childhood survivors have shown conflicting results. ${ }^{2-4-11}$ Reduced lung volumes ${ }^{28}$ and reduced expiratory flows ${ }^{3}$ or both $^{9}$ have been reported, but others have reported no spirometric abnormalities. ${ }^{4} 1011$ Only one study ${ }^{3}$ used matched controls. An increase in respiratory symptoms and intolerance of exercise have been reported, ${ }^{38}$ but others have not found any continuing morbidity. ${ }^{10}{ }^{11}$ Subjective reporting of impaired exercise performance has been noted in some studies. ${ }^{3689}$ An uncontrolled study found normal values of oxygen uptake, ${ }^{10}$ but a recent study has compared a group of $\mathrm{CDH}$ survivors with controls and shown reduced maximum oxygen consumption $\left(\dot{\mathrm{V}}_{2} \mathrm{max}\right)$ in index cases. ${ }^{12}$ Growth in survivors of $\mathrm{CDH}$ has been studied in less detail, although there is a suggestion that such children may be underweight. ${ }^{12}{ }^{13}$ Our objective was therefore to examine growth, lung function, and exercise performance in a cohort of Sheffield survivors of CDH repair compared with matched controls.

\section{Methods}

SUBJECTS

Ethical permission for the study was granted by the South Sheffield research ethics committee.

Between 1978 and 1988, 48 patients were referred to the surgical neonatal unit in Sheffield with a diagnosis of CDH. Nineteen $(79 \%)$ of 24 survivors were recruited. One patient had moved away and four were untraceable. Fifteen $(79 \%)$ presented on the first day of life, $16(84 \%)$ were left sided, and all survivors were operated on within 48 hours of presentation. Median duration of ventilation was four days (range one to 19). All were term deliveries. Mean birth weight of $3.27 \mathrm{~kg}$ (range 2.9 to 3.84 ). Four had recurrences requiring further surgery. Two had other surgery, one for a duodenal perforation and one for gastrooesophageal reflux. One child had associated hydrocephalus and one had an atrial septal defect and pulmonary hypertension. Eighteen survivors were white and one was black Afro-Caribbean. Eleven were male. Mean age was 11.51 years (range 7.3 to 16.9) for survivors and 11.55 years (7.23 to 16.74 ) for controls. The control subjects were identified by a head teacher and were matched for age, gender, and race. A control was found for each survivor. Survivors and matched controls were studied on the same day under the same conditions.

Detailed auxology was performed by an experienced measurement technician. Standing and sitting heights were measured with a 
Harpenden stadiometer, and body mass index (BMI) was calculated as weight/height ${ }^{2}$. Pubertal staging was performed using Tanner's criteria. $^{14}$

RESPIRATORY SYMPTOMS AND SPIROMETRY

All subjects were examined and asked to complete a standardised respiratory questionnaire with the help of their parent/guardian. The questionnaire contained the core questions from the ISAAC (international study of asthma and allergy in childhood) questionnaire. ${ }^{15}$

Spirometry (maximum expiratory flowvolume loops) and lung volume measurements (helium dilution) were made using a Morgan eight litre "TLC test" rolling seal dry spirometer. Each child received a short period of instruction from an experienced technician immediately before testing; tests were performed with the children standing and wearing nose clips. Forced expiratory variables (forced expiratory volume in one second $\left(\mathrm{FEV}_{1}\right)$, forced vital capacity (FVC)) were determined from the best of three reproducible manoeuvres ${ }^{16}$ and expressed as percentages of predicted values for height based on data from Sheffield schoolchildren. ${ }^{17}$ Helium dilution volumes were expressed as percentages of predicted values for height based on published norms. ${ }^{18}$

EXERCISE TESTING

Exercise was performed on an electrically braked cycle ergometer (Bosch ERG 550, Berlin, Germany). Electronic control of the ergometer ensures that the work intensity is independent of pedal speed. Respiratory gases were monitored on a breath by breath basis using a respiratory mass spectrometer (Marquette MGA1 100, Marquette Electronics, Milwaukee, USA) and turbine flow meter (Interface Associates, Ventilation Measurement Module model VMM-2A). Cardiac frequency was recorded using a three lead electrocardiograph. The "First Breath v2.0" software (Marquette Electronics) integrated the signals from the mass spectrometer, flow meter, and electrocardiograph to provide on-line determination of metabolic and cardiorespiratory variables. The software also supplied the work rate control signal for the cycle ergometer.

Exercise protocols

Each subject performed a continuous incremental ramp protocol to maximum volitional exertion on the cycle ergometer in order to assess aerobic function. ${ }^{19}$ Before starting the test, seat height and handlebars were individually adjusted and each subject was allowed a period of familiarisation with the respiratory apparatus and ergometer. To account for the variation in age, height, and weight of the test population, three different ramp protocols were used to ensure that the test would terminate after approximately 10 minutes of cycling. ${ }^{20}$ Standing height was used as the criterion for determining the protocol to be undertaken. ${ }^{21}$ We used a ramp protocol of 10 $\mathrm{W} / \mathrm{min}$ for a height below $125 \mathrm{~cm}, 15 \mathrm{~W} / \mathrm{min}$ for heights between 125 and $150 \mathrm{~cm}$, and 20
$\mathrm{W} / \mathrm{min}$ for taller children. Where the height of the index and matched control fell into different protocol categories, the protocol appropriate to the shorter child was used. The index and control subjects undertook the test on the same day, one after the other in a randomised order. All protocols began with unloaded cycling, after which the workload increased continuously at a rate equivalent to 10,15 , or $20 \mathrm{~W} / \mathrm{min}$. Subjects were asked to maintain a pedal frequency of 60 revolutions per minute throughout the exercise. Strong verbal encouragement was used to motivate the subject during the latter stages of the test, which terminated when the subject was unwilling or unable to continue. ${ }^{22}$

Analysis of exercise data

The final work rate was taken as the highest work rate achieved at test termination. Breath by breath respiratory data and heart rate were all expressed as 30 second averages. ${ }^{23}$ The following variables were recorded at peak exercise: oxygen uptake $\left(\dot{\mathrm{V}}_{2}\right)$, carbon dioxide production $\left(\dot{\mathrm{V}}_{\mathrm{CO}}\right)$, respiratory exchange ratio (RER), minute ventilation (Ve), tidal volume $(\mathrm{Vt})$, respiratory frequency (rf), fractional end tidal oxygen $\left(\mathrm{FETO}_{2}\right)$ and fractional end tidal carbon dioxide $\left(\mathrm{FETCO}_{2}\right)$. The time taken for inspiration ( $\mathrm{Ti}$ ) was calculated as a percentage of the total breath duration (Ttot). The tidal volume inspired ( $\mathrm{Vi}$ ) and the tidal volume expired (Ve) were expressed as a ratio of the time taken for inspiration and expiration respectively ( $\mathrm{Vi} / \mathrm{Ti}$ and $\mathrm{Ve} / \mathrm{Te}$ ). The $\dot{\mathrm{V}}_{2}$ plateau was defined as an increase in $\dot{\mathrm{V}}_{2}$ during the final stages of exercise of less than 2 SD below the mean increases in $\dot{\mathrm{V}}_{2}$ during previous submaximal work rates. ${ }^{24}$ Subjects were judged not to have made an adequate effort if they failed to achieve any one of the following criteria: (1) RER > 1; (2) maximum heart rate within 2 SD of that predicted ${ }^{21}$; (3) plateau achieved in $\dot{\mathrm{V}}_{2}$.

ACTIVITY AND ATTITUDE QUESTIONNAIRES

Habitual activity questionnaire

The activity questionnaire was designed to identify, by interview, the amount of time spent by each child in low, moderate, and high intensity physical activities during the seven days preceding the visit to the laboratory. ${ }^{25} \mathrm{~A}$ weekly activity score was calculated by summing the scores from each period of activity. The scores ranged from 1 to 7 , where a score of 1 was assigned to a low intensity exercise period of less than 20 minutes and a score of 7 denoted a high intensity exercise for more than 20 minutes.

Perception and attitude questionnaires

A five point Likert scale ${ }^{26}$ was used to establish the children's perception of personal fitness when compared to their peers. The scope of the response was limited to those walking, running, or cycling activities which had been identified in the habitual activity questionnaire. A similar five point scale was used to determine the child's general attitude towards exercise. 
Table 1 Growth data in 18 children surviving $C D H$ repair and matched controls

\begin{tabular}{llll}
\hline & CDH & Controls & Differences (index-control) \\
\hline $\begin{array}{l}\text { Height SD } \\
\text { score }\end{array}$ & $0.19(-0.23$ to +0.60$)$ & $-0.04(-0.46$ to +0.38$)$ & $+0.22(-0.25$ to +0.70$)$ \\
Weight SD & $-0.30(-0.99$ to +0.40$)$ & $-0.18(-0.71$ to +0.33$)$ & $-0.11(-0.73$ to +0.51$)$ \\
$\quad$ score & & & \\
BMI SD score & $-0.61(-1.44$ to +0.23$)$ & $-1.36^{\star}(-2.01$ to -0.7$)$ & $+0.75(-0.115$ to +1.62$)$ \\
OFC SD score & $-0.74^{\star}(-1.4$ to -0.07$)$ & $-0.22(-0.72$ to +0.28$)$ & $-0.55(-1.34$ to +0.24$)$ \\
$\begin{array}{l}\text { Sitting height } \\
\text { SD score }\end{array}$ & $-0.72(-0.69$ to +0.54$)$ & $-0.56(-1.20$ to +0.08$)$ & $-0.49(-0.11$ to +1.09$)$ \\
\hline
\end{tabular}

Values shown are mean (95\% confidence interval). BMI = body mass index; OFC = occipitofrontal head circumference.

${ }^{\star}$ Significantly different from reference values $(\mathrm{p}<0.05)$.

Table 2 Reported respiratory morbidity in children surviving CDH repair and matched controls

\begin{tabular}{lcc}
\hline Symptoms & Survivors & \multicolumn{1}{l}{ Controls } \\
\hline Ever wheezed & $7(37 \%)$ & $5(26 \%)$ \\
Wheezed in last 12 months & $6(32 \%)$ & $3(16 \%)$ \\
Severe wheeze limiting speech & $1(5 \%)$ & $1(5 \%)$ \\
Persistent cough more than weekly & $1(5 \%)$ & $2(11 \%)$ \\
Night cough more than weekly & $2 / 18(11 \%)$ & $1 / 19(6 \%)$ \\
Asthma ever & $8(42 \%)$ & $3(16 \%)$ \\
Current asthma & $5(26 \%)$ & $4(21 \%)$ \\
Exercise induced wheeze? & $8(42 \%)$ & $4(21 \%)$ \\
Sometimes & 5 & 4 \\
Most times & 3 & 0 \\
Ever had eczema & $6(31 \%)$ & $4(23 \%)$ \\
Ever had hayfever & $3(17 \%)$ & $2(11 \%)$ \\
Family history of atopy & $10(53 \%)$ & $11(58 \%)$ \\
\hline
\end{tabular}

Table 3 Percentage predicted lung function in children surviving CDH repair and matched controls

\begin{tabular}{lclc}
\hline & Index & Control & Differences (index-control) \\
\hline TLC & $89.3(83.3$ to 95.4$)$ & $94.1(90.0$ to 98.2$)$ & $-4.8(-12.0$ to +2.4$)$ \\
FRC & $94.7(86.0$ to 103.4$)$ & $97.6(89.4$ to 105.5$)$ & $-2.9(-16.0$ to +10.2$)$ \\
RV & $101.0(77.9$ to 124.2$)$ & $77.0(62.5$ to 91.6$)$ & $+24.0(-3.0$ to +51.0$)$ \\
RV/TLC & & $16.2(13.0$ to 19.3$)$ & $+5.3^{\star}(+0.20$ to +10.5$)$ \\
$\quad($ absolute ratio) & $21.5(17.5$ to 26.3$)$ & & \\
FVC & $84.7(78.8$ to 90.6$)$ & $96.5(91.1$ to 101.9$)$ & $-11.8+(-19.1$ to -4.5$)$ \\
$\mathrm{FEV}_{1}$ & $78.7(72.5$ to 84.8$)$ & $92.3(86.7$ to 97.9$)$ & $-13.6+(-22.1$ to -5.2$)$ \\
$\mathrm{FEF}_{25-75}$ & $69.0(55.9$ to 82.1$)$ & $83.7(76.5$ to 90.9$)$ & $-14.7^{\star}(-27.9$ to -1.6$)$ \\
$\mathrm{FEF}_{25}$ & $80.5(69.2$ to 91.9$)$ & $95.4(87.1$ to 103.7$)$ & $-14.9^{\star}(-29.5$ to -0.3$)$ \\
$\mathrm{FEF}_{50}$ & $77.4(64.8$ to 90.1$)$ & $90.9(82.2$ to 99.7$)$ & $-13.5(-27.5$ to +0.5$)$ \\
$\mathrm{FEF}_{75}$ & $83.0(68.7$ to 97.3$)$ & $88.0(78.0$ to 97.9$)$ & $-5.0(-19.7$ to +9.7$)$ \\
\hline
\end{tabular}

Values shown are mean (95\% confidence interval). TLC $=$ total lung capacity; FRC $=$ functional residual capacity; $\mathrm{RV}=$ residual volume; $\mathrm{FVC}=$ forced vital capacity; $\mathrm{FEV}_{1}=$ forced expiratory volume in 1 second; FEF $=$ forced expiratory flow.

$\mathrm{tp}<0.01,{ }^{\star} \mathrm{p}<0.05$ index cases compared with controls (paired $t$ test).

Table 4 Forced expiratory flows (FEF) corrected for FVC in children surviving CDH repair and matched controls

\begin{tabular}{lllr}
\hline & Index & Control & Differences (index-control) \\
\hline $\mathrm{FEF}_{25}$ & $1.67(1.37$ to 1.96$)$ & $1.69(1.53$ to 1.85$)$ & $-0.03(-0.34$ to +0.29$)$ \\
$\mathrm{FEF}_{50}$ & $1.24(0.98$ to 1.50$)$ & $1.24(1.11$ to 1.37$)$ & $0.00(-0.21$ to +0.21$)$ \\
$\mathrm{FEF}_{75}$ & $0.68(0.52$ to 0.84$)$ & $0.61(0.53$ to 0.69$)$ & $+0.07(-0.06$ to +0.20$)$ \\
$\mathrm{FEF}_{25-75}$ & $0.98(0.75$ to 1.21$)$ & $1.02(0.91$ to 1.12$)$ & $-0.04(-0.23$ to +0.15$)$ \\
\hline
\end{tabular}

Units are FVC/sec. Values shown are mean (95\% confidence interval).

There are no significant differences.

STATISTICAL ANALYSIS

Standard deviation scores for height, weight, sitting height, head circumference, and BMI were calculated from reference data. ${ }^{27}$ Continuous data from survivors and controls were compared using paired $t$ tests (two tailed). Questionnaire data were tabulated and described as the numbers were too few for statistical comparison. Simple regression analyses or two group $t$ tests were used to determine relations between perinatal variables and lung function.

Perception and attitude (Likert) scores were compared categorically. Pearson product moment correlation was used to describe the relation between the activity scores and $\dot{\mathrm{V}}_{2} \max$ $(\mathrm{ml} / \mathrm{kg} / \mathrm{min})$. The study was estimated to have a $90 \%$ power of detecting a difference of $10 \%$ in percentage predicted FVC at 5\% significance, using 19 pairs.

\section{Results}

GROWTH

Six of the index cases had a chest deformity, consisting of pectus excavatum in four, of whom two had ventral asymmetry; Harrison's sulci and asymmetry in one case, and asymmetry alone in one case. An index case was receiving oxandrolone for pubertal delay, and he and his control were excluded from growth analysis. Data on head circumference were missing for one index case. Fortuitously, each index case was at the equivalent pubertal stage to his or her matched control. Growth data for the index and control groups are shown in table 1. There were no significant differences between the two groups. The head circumference standard deviation score for index cases was significantly below the normal value of 0 $(\mathrm{p}<0.05)$, as was the BMI score for the control children $(\mathrm{p}<0.01)$.

RESPIRATORY SYMPTOMS AND SPIROMETRY

Respiratory questionnaires were correctly completed and returned by 18 survivors and 17 controls. The results are shown in table 2 . About twice as many of the index cases reported respiratory symptoms, but the numbers were too small to draw statistical conclusions. The spirometry results are shown in table 3. The FEV 1 and FVC were significantly lower in the index cases $(p<0.01)$, but no significant difference was seen in total lung capacity (TLC). The control group residual volume (RV) was significantly lower than predicted but did not differ significantly from that of the index cases. Neither the side of the hernia nor the duration of neonatal ventilation significantly predicted subsequent $\mathrm{FEV}_{1}$ or FVC. Although early $\left(\mathrm{FEF}_{25}\right)$ and mid $\left(\mathrm{FEF}_{25-}\right.$ 75) forced expiratory flow rates were significantly lower in index cases, this difference disappeared when the flow rates were expressed in units of $\mathrm{FVC} / \mathrm{sec}$ (table 4). This indicates that the observed differences in flows were due to lower functional lung volumes rather than airway obstruction

EXERCISE PERFORMANCE

Of the 19 pairs of children tested, three were excluded from analyses. One control subject was too small to cycle and two index subjects (one of whom had a heart defect) failed to make an adequate effort. There were no significant differences between the index children and their controls in any of the cardiopulmonary variables measured at exhaustion (table 5). All the children achieved RER > 1 , while $94 \%$ of index children and $88 \%$ of controls achieved a maximum heart rate within 2 SD of that predicted. Only $38 \%$ of index children and $13 \%$ of controls achieved a plateau of oxygen uptake. Ventilation at maximum aerobic exercise was significantly different between the two groups. The signifi- 
Table 5 Peak cardiorespiratory responses to the cycle ergometer exercise ( $n=16$ pairs)

\begin{tabular}{lclllll}
\hline & Index & & \multicolumn{3}{c}{ Control } & \multicolumn{2}{c}{ Differences (index-control) } \\
\hline Final work rate (W) & 136 & $(111-161)$ & 147 & $(122-171)$ & -11 & $(-24$ to +3$)$ \\
$\dot{\mathrm{V}} \mathrm{o}_{2} \mathrm{max}(\mathrm{ml} / \mathrm{kg} / \mathrm{min})$ & 40.1 & $(36.8-43.4)$ & 42.2 & $(38.5-45.8)$ & -2.1 & $(-4.8$ to +0.7$)$ \\
$\dot{\mathrm{V}}$ & $2 \mathrm{max}(1 / \mathrm{min})$ & 1.52 & $(1.26-1.78)$ & 1.66 & $(1.41-1.91)$ & $-0.14(-0.31$ to +0.03$)$ \\
$\mathrm{RER}$ & 1.13 & $(1.10-1.17)$ & 1.14 & $(1.11-1.18)$ & $-0.01(-0.03$ to +0.01 \\
$\mathrm{HR}$ (beats $/ \mathrm{min})$ & 197 & $(191-202)$ & 191 & $(184-198)$ & +6 & $(-2$ to +13$)$ \\
\hline
\end{tabular}

Values shown are mean (95\% confidence interval). $\dot{V}_{2} \max =$ maximum oxygen consumption; RER, respiratory exchange ratio; HR, heart rate.

No differences are significant.

Table 6 Average ventilatory variables at exhaustion for index and control groups ( $n=16$ pairs)

\begin{tabular}{|c|c|c|c|}
\hline & Index & Control & Differences (index-control) \\
\hline $\mathrm{Ve}(1 / \mathrm{min})$ & $56 \quad(47.5-64.5)$ & $60 \quad(52-68)$ & $-4.3 \quad(-9.0$ to +0.4$)$ \\
\hline $\mathrm{Vt}(\mathrm{l})$ & $1.04(0.81-1.27)$ & $1.23(1.01-1.45)$ & $-0.19^{\star}(-0.05$ to -0.32$)$ \\
\hline rf (breaths/min) & $56 \quad(51-61)$ & $50 \quad(46-54)$ & $5.9^{\star} \quad(+0.8$ to +11.1$)$ \\
\hline $\mathrm{Ti} / \mathrm{Tb}(\%)$ & $50 \quad(48-52)$ & $52 \quad(51-53)$ & $(-3.5$ to +0.3$)$ \\
\hline $\mathrm{Vi} / \mathrm{Ti}(1 / \mathrm{s})$ & $1.59(1.33-1.85)$ & $1.65(1.42-1.88)$ & $-0.07 \quad(-0.2$ to +0.06$)$ \\
\hline $\mathrm{Ve} / \mathrm{Te}(1 / \mathrm{s})$ & $1.51(1.29-1.73)$ & $1.68(1.45-1.91)$ & $-0.17^{\star}(-0.33$ to -0.01$)$ \\
\hline
\end{tabular}

Values shown are mean ( $95 \%$ confidence interval). $\mathrm{Ve}=$ minute ventilation; $\mathrm{Vt}=$ tidal volume; $\mathrm{rf}$ $=$ respiratory frequency; $\mathrm{Ti} / \mathrm{Tb}=$ inspiratory percentage of breath cycle; $\mathrm{Vi} / \mathrm{Ti}=$ mean inspiratory flow; $\mathrm{Ve} / \mathrm{Te}=$ mean expiratory flow.

${ }^{\star} \mathrm{p}<0.05$.

cantly reduced $\mathrm{Vt}$ in the $\mathrm{CDH}$ group was partially compensated by an increased frequency of breathing (table 6).

In spite of the differences in ventilation apparent between the CDH survivors and their controls there was no difference in the alveolar gas concentrations at maximum exercise. Mean (SD) $\mathrm{FETO}_{2}$ values for index and control children were $0.16(0.01)$ and $0.16(0.01)$, while $\mathrm{FETCO}_{2}$ values were $0.054(0.006)$ and 0.053 (0.005) respectively.

ACTIVITY AND ATTITUDE QUESTIONNAIRES The activity scores were similar for the two groups with mean scores of 75 (43) and 79 (43) for index and control children respectively. There was a significant positive correlation $(r=$ $0.53, \mathrm{p}<0.01)$ for the group as a whole between activity scores and $\mathrm{VO}_{2} \max$. When completing the Likert scale on attitudes to exercise, none of the children reported that they disliked exercise. Sixty three per cent of the index children and $94 \%$ of the controls enjoyed exercising. With regard to self perception of fitness, the index children perceived their own fitness to be worse than the control group with $63 \%$ (95\% confidence interval $39 \%$ to $87 \%$ ) of the controls and $25 \%$ ( $5 \%$ to $45 \%$ ) of the index subjects considering themselves fitter than their friends $(p<0.05)$.

\section{Discussion}

GROWTH

There are few data on growth in survivors of $\mathrm{CDH}$. Zaccara et $a l^{13}$ found normal height attainment but lower than expected weight in a subgroup of survivors. In survivors of extracorporeal membrane oxygenation, growth and nutrition was found to be progressively impaired over the first two years in $\mathrm{CDH}$ infants compared to those with other pathology, ${ }^{12}$ but these children had more severe disease than our cohort, and included several with long term oxygen dependency and major feeding problems. Our subjects showed no significant growth impairment compared with their con- trols, and although both groups had one growth variable which differed from reference norms, in neither case was this of sufficient magnitude to suggest a biological significance.

RESPIRATORY SYMPTOMS AND SPIROMETRY

$\mathrm{CDH}$ is known to cause a varying degree of pulmonary hypoplasia, ${ }^{29}$ and survivors have been shown to have persisting abnormalities in ventilation and perfusion in both lungs, ${ }^{37}$ which might be expected to impair exercise performance. Nevertheless, pulmonary ventilation is not considered a limiting factor to aerobic power in healthy children. ${ }^{30}$ In view of the reduced numbers of airway divisions in this condition, it is likely that reduction in lung volumes would persist in survivors, but there is less reason intuitively to expect airway obstruction. Although the spirometric abnormalities reported in previous work have been inconsistent, most studies were uncontrolled and have often involved a relatively small selected group from among a total population of $\mathrm{CDH}$ survivors.

Our study of $79 \%$ of survivors using matched controls has shown that the predominant spirometric impairment is in functional lung volumes. The apparent reductions in expiratory flows were merely a reflection of the reduction in FVC, and were eliminated by correcting for lung size. It is surprising that a concomitant reduction in TLC was not seen. Recent work from the Netherlands ${ }^{31}$ has also observed reduced $\mathrm{FEV}_{1}$ and normal TLC, with an increase in RV/TLC, although FVC results were not given. The index cases in our study also appeared to have a higher RV/TLC ratio, despite the lack of airway dysfunction, and it is possible that there is a permanent loss of lung elasticity leading to a degree of emphysema and smaller functional lung volumes.

Previous findings of emphysema on $x$ ray would support this. ${ }^{25}$ Similar findings of increased RV/TLC without airway obstruction have been observed in very low birthweight survivors. ${ }^{32}$ They may represent gas trapping in emphysematous or overdistended alveoli, possibly due to loss of elasticity.

Mild increases in RV/TLC associated with normal airway function appear not to be associated with symptoms. ${ }^{10}$ We observed more reported symptoms in index cases, but the numbers available for study preclude firm conclusions being drawn. The overall impact of the spirometric abnormalities in our cases did not appear to be severe.

EXERCISE PERFORMANCE, ACTIVITY, AND ATTITUDES

Our study had $90 \%$ power to detect a difference of $12.5 \%$ in mean $\dot{\mathrm{V}}_{2} \mathrm{max} / \mathrm{kg}$ at $5 \%$ significance. We did not detect a significant difference in $\dot{\mathrm{V}}_{2} \mathrm{max} / \mathrm{kg}$, although there were differences in the mechanics of exercise performance, evidenced by shallower, more rapid breathing in index children to achieve similar maximum minute ventilation. These findings are compatible with the reduced functional lung volumes which we have demonstrated in the index children. Inspiration was 
attenuated more noticeably, which may have increased the energy cost of breathing slightly. The different breathing patterns of the two groups did not affect alveolar gas concentrations. Vanamo et al showed that diffusing capacity was normal in $\mathrm{CDH}$ patients, ${ }^{9}$ excluding the possibility of a significant disturbance in gas exchange at the level of the alveolar membrane.

The average aerobic power $\left(\dot{\mathrm{V}}_{2} \mathrm{max}\right)$ achieved by both groups is typical for normal children aged between 6 and 18 years. Armstrong and Davies ${ }^{30}$ found a weight corrected $\mathrm{V}_{2}$ max of between 38 and $42 \mathrm{ml} / \mathrm{kg} /$ min. The European Paediatric Work Physiology Group recommend a minimum aerobic power of $35 \mathrm{ml} / \mathrm{kg} / \mathrm{min}$ for boys and $30 \mathrm{ml} / \mathrm{kg} /$ min for girls. ${ }^{33}$ Only one child from the $\mathrm{CDH}$ group and no children in the control group fell below the recommended level of aerobic power so could be classified as unfit. The aerobic power demonstrated by the children surviving $\mathrm{CDH}$ repair and their controls means that on average these children should be capable of continuous running at speeds up to 10 $\mathrm{km} /$ hour. ${ }^{34}$ The small difference of $2 \mathrm{ml} / \mathrm{kg} / \mathrm{min}$ between the groups has very little functional significance in terms of exercise or sporting performance.

A vexed question in the study of exercise in children is whether a maximum level has been reached. This study identifies specific criteria for maximum oxygen uptake. Strong verbal encouragement was given to each child but because of the way in which the cycle ergometer increases the resistance once the cadence drops, it is difficult for a child to have enough leg strength to resume the exercise if the pedal frequency becomes too low. In order to quantify maximum effort, either heart rate or respiratory exchange ratio can be used. Another criterion is a plateau in oxygen uptake, even though the work rate is still increasing, although some investigators ${ }^{24}$ argue strongly against the use of the plateau method of defining maximum effort in children. As expected ${ }^{33}$ only a minority of children in this study showed a plateau, but more $\mathrm{CDH}$ survivors were in this category than control children. Similarly the group of index children reached higher heart rate at exhaustion (197 (9.8) beats/min) than the control group (191 (13.1)), which suggests that it is likely that maximum effort was obtained. Average respiratory exchange ratios of 1.13 and 1.14 for $\mathrm{CDH}$ and control groups, respectively, are again indicative of maximum effort and support the view that strong verbal encouragement is required if maximum values are to be achieved. ${ }^{30}$

Of the two most recent studies, Vanamo et al described a subjective intolerance in $13 \%$ of survivors, ${ }^{9}$ many of whom were adults when investigated. Zaccara et al studied 15 children of similar mean age to the Sheffield children (11 years 3 months) by exercising them to exhaustion on a treadmill, ${ }^{13}$ using a walking/ running protocol. They found that their $\mathrm{CDH}$ patients had significantly lower aerobic capacities than their control group. They explained the low $\dot{\mathrm{V}}_{2} \max$ of $31 \mathrm{ml} / \mathrm{kg} / \mathrm{min}$ by the low exercise involvement of some of the $\mathrm{CDH}$ group. This was due in part to the extensive medical screening required by Italian law before children are permitted to participate in sport. Other explanations may lie in the fact that the Italian children were underweight for their age and also that they may not have reached maximum effort. On average, maximum heart rates of only 187 beats/min were recorded by these investigators. A similar problem occurred in the study of Freyschuss et al, where maximum mean heart rates of 186 beats/min and mean RERs of 0.98 were reported. ${ }^{10}$ It is unlikely that the subjects studied by these workers achieved maximum effort.

One follow up investigation of $\mathrm{CDH}$ survivors has linked physical fitness with activity levels, ${ }^{13}$ showing significant differences in $\dot{\mathrm{V}}_{2} \max (1 / \mathrm{min})$ between $\mathrm{CDH}$ patients who were sports participants and those who were sedentary. For the Sheffield children, no difference could be shown between the children surviving $\mathrm{CDH}$ and their controls. This may be partially explained by the fact that the children and their controls came from the same school class and experienced the same physical education curriculum. Other investigators of healthy children have shown a tenuous link between physical activity and physical fitness $^{35} 36$ and have concluded that inherited factors are more important in determining $\dot{\mathrm{V}}_{2} \max$ than environmental factors. It was interesting therefore that both the control group and the $\mathrm{CDH}$ survivors showed significant positive correlations between $\dot{\mathrm{V}}_{2}$ max (expressed as $\mathrm{ml} / \mathrm{kg} / \mathrm{min}$ ) and activity scores $(r$ $=0.52$ and $r=0.64$ for controls and $\mathrm{CDH}$ group, respectively).

We considered how CDH survivors viewed their personal fitness. When asked to rate themselves in terms of fitness and also in terms of their attitude to exercise, the children surviving $\mathrm{CDH}$ tended to consider themselves as less fit than their peers and they also had a more negative attitude towards exercise in general. This is an interesting finding in view of the previous reports of subjective exercise intolerance, some of which may have been related to attitude and perception rather than to objective impairment. If the children surviving $\mathrm{CDH}$ repair are to achieve their full potential, it is essential that parents, teachers, and doctors encourage fuller participation in an active lifestyle, not only because it improves the general health of children ${ }^{37}$ but also because the exercise habits of childhood are likely to be maintained into adult life. ${ }^{38}$

The neonatal management of congenital diaphragmatic hernia is changing. Preoperative stabilisation identifies those infants most likely to do well. In addition, advances such as high frequency oscillation and extracorporeal membrane oxygenation offer survival to those with more severe lung hypoplasia. ${ }^{12} 39$ Our cohort survived a period of less sophisticated neonatal care, and is likely to represent a milder form of $\mathrm{CDH}$ than those who might be reported in the future. However, we can conclude that children with $\mathrm{CDH}$ who do not require such intensive intervention can be expected to have reduced 
functional lung volumes but normal growth and relatively minor functional effects. In addition, the degree of exercise impairment observed in the $\mathrm{CDH}$ survivors has little if any functional significance. In the absence of associated abnormalities the children should be expected to achieve normal exercise function. We consider it unlikely, therefore, that children surviving $\mathrm{CDH}$ are adversely affected to any great extent by their condition, and we suggest that they should be encouraged to participate more fully in all forms of exercise and sport.

We wish to thank Mr J A S Dickson and Mr A E MacKinnon (consultant paediatric surgeons) and all the head teachers involved for their help in identifying the survivors and their controls. The study was funded by the CHRIS fund of the Sheffield Children's Hospital.

1 Sweed Y, Puri P. Congenital diaphragmatic hernia: influence of associated malformations on survival. Arch Dis Child 1993;69:68-70.

2 Chatrath R, El Shafie M, Jones R. Fate of hypoplastic lungs after repair of congenital diaphragmatic hernia. Arch Dis after repair of conge
Child $1971 ; 46: 633-5$.

3 Falconer A, Brown R, Helms P, et al. Pulmonary sequelae in survivors of congenital diaphragmatic hernia. Thorax 1990 45:126-9.

4 Wohl M, Griscom N, Strieder D, et al. The lung following repair of congenital diaphragmatic hernia. $\mathcal{F}$ Pediatr 1977;90:405-14.

5 Reid I, Hutcherson R. Long term follow up of patients with congenital diaphragmatic hernia. F Pediatr Surg 1976;11 939-42.

6 Delepoulle F, Martinot A, Leclerc F, et al. Devenir a long terme des hernies diaphragmatiques congénitales. Etude de 17 patients. Arch Fr Pediatr 1991;48:703-7.

7 Jeandot R, Lambert B, Brendel A, et al. Lung ventilation and perfusion scintigraphy in the follow up of repaired congenital diaphragmatic hernia. Eur f Nucl Med 1989;15:591-6.

8 Wischermann A, Holscchneider A, Hubner U. Long term follow up of children with diaphragmatic hernia. Eur $\mathcal{F}$ follow up of children with

9 Vanamo K, Rintala R, Sovijarvi A, et al. Long-term pulmonary outcome in survivors of congenital diaphragmatic defects. F Pediatr Surg 1996:31:1096-100.

10 Freyschuss U, Lannergren K, Frenckner B. Lung function after repair of congenital diaphragmatic hernia. Acta Paediatr Scand 1984;73:589-93.

11 Kerr A. Lung function in children after repair of congenital diaphragmatic hernia. Arch Dis Child 1977;52:902-3.

12 Zaccara A, Turchetta A, Calzolari A, et al. Maximal oxygen consumption and stress performance in children operated on for congenital diaphragmatic hernia. $f$ Pediatr Surg 1996;31:1092-5.

13 Van Meurs K, Robbins S, Reed V, et al. Congenital diaphragmatic hernia: long-term outcome in neonates treated with extracorporeal membrane oxygenation. 7 Pediatr 1993;122:893-9.

14 Tanner J. Growth at adolescence. 2nd Ed. Oxford: Blackwell Scientific, 1962:28-39.

15 Asher M, Keil U, Anderson H, et al. International study of asthma and allergies in childhood (ISAAC): rationale and asthma and allergies in childhood (ISA
methods. Eur Resp f 1995;8:483-91.
16 Quanjer P, Tammelin G, Cotes J, et al. Lung volumes and forced ventilatory flows. Report of the working party on standardisation of lung function tests. European Coal and Steel Community. Eur Resp f 1993;6(suppl 16):5-40.

17 Primhak R. The effect of growth, environment and ethnic origin on lung function in children. Sheffield: Sheffield University, 1986. [MD thesis.]

18 Cotes J. Lung function. Assessment and application in medicine. 4th Ed. London: Blackwell Scientific, 1979.

19 Whipp B, Davis J, Torres F. A test to determine parameters of aerobic function during exercise. $\mathcal{F}$ Appl Physiol 1981;50: $217-21$

20 Wasserman K, Hansen J, Sue D, et al. Principles of exercise testing and interpretation. Philadelphia: Lea and Febiger, 1987.

21 Godfrey S. Exercise testing in children: applications in health and disease. Philadelphia: WB Saunders, 1974.

22 Armstrong N, Williams J, Balding J, et al. The peak oxygen uptake in British children with reference to age, sex and sexual maturity. Eur f Appl Physiol Occup Physiol 1991;62: sexual mat

23 Zeballos RJ, Weisman IM. Behind the scenes of cardiopulmonary exercise testing. Clin Chest Med 1994;15:193-213.

24 Rowland T, Cunningham L. Oxygen uptake plateau during maximal treadmill exercise in children. Chest 1992;101: $485-9$.

25 Cale L. Self-report measures of children's physical activity: recommendations for future development and a new alternative measure. Health Educ f 1994;53:439-53.

26 Oppenheim A. Questionnaire design and attitude measurement. London: Heinemann, 1966.

27 Freeman J, Cole T, Chinn S, et al. Cross sectional stature and weight reference curves for the UK, 1990. Arch Dis Child 1995;73:17-24.

28 Cole T, Freeman J, Preece M. Body mass index reference curves for the UK, 1990. Arch Dis Child 1995;73:25-9.

29 Hislop A, Reid L. Persistent hypoplasia of the lung after repair of congenital diaphragmatic hernia. Thorax 1976;31: $450-5$.

30 Armstrong N, Davies B. The metabolic and physiological responses of children to exercise and training. Phys Educ Rev 1984;2:90-105.

31 Ijsselstijn $\mathrm{H}$, Tibboel $\mathrm{D}$, Hop W, et al. Long-term pulmonary sequelae in children with congenital diaphragmatic hernia. Am f Resp Crit Care Med 1997;155:174-80.

32 MacKenzie C. Very low birth weight survivors: functional status at 8-10 years. Sheffield: Sheffield University, 1992. [MD thesis.]

33 Armstrong N, McManus A, Welshman J. Children's aerobic fitness. Brf Phys Educ 1994:9-11.

34 Astrand P-O, Rodahl K. Textbook of work physiology. 3rd Ed. Singapore: McGraw-Hill, 1986:362.

35 Morrow JJ, Freedson P. Relationship between physical activity and aerobic fitness in adolescents. Pediatr Exerc Sci 1994;6:315-29.

36 Pate R, Dowda M, Ross J. Associations between physical activity and physical fitness in American children. Am $\mathcal{F}$ Dis Child 1990;144:1123-9.

37 Baranowski T, Bouchard C, Bar Or O, et al. Assessment, prevalence, and cardiovascular benefits of physical activity and fitness in youth. Med Sci Sports Exerc 1992;24:S237-

38 Kemper H. Growth, health and fitness of teenagers. Longitudinal research in international perspective. Med Sports Sci 1985;20:1-11.

39 Nakayama D, Motoyama E, Mutich R, et al. Pulmonary function in newborns after repair of congenital diaphragmatic hernia. Pediatr Pulmonol 1991;11:49-55. 